\title{
Electroacupuncture Promotes Remyelination and Alleviates Cognitive Deficit Via Promoting OPC Differentiation in A Rat Model of Subarachnoid Hemorrhage
}

\section{Yingwen Wang}

The First Affiliated Hospital of Chongqing Medical University

\section{Xiaomin Yang}

The First Affiliated Hospital of Chongqing Medical University

\section{Yunchuan Cao}

The First Affiliated Hospital of Chongqing Medical University

\section{Xiaoguo Li}

The First Affiliated Hospital of Chongqing Medical University

\section{Rui Xu}

The First Affiliated Hospital of Chongqing Medical University Jin Yan

The First Affiliated Hospital of Chongqing Medical University

\section{Zongduo Guo}

The First Affiliated Hospital of Chongqing Medical University

\section{Shanquan Sun}

Chongqing Medical University

\section{Xiaochuan Sun}

The First Affiliated Hospital of Chongqing Medical University

\section{Yue Wu ( $\nabla$ wuyuedt@163.com )}

The First Affiliated Hospital of Chongqing Medical University

\section{Research Article}

Keywords: SAH, Electroacupuncture, Remyelination, Cognitive deficit

Posted Date: March 9th, 2022

DOI: https://doi.org/10.21203/rs.3.rs-1305516/v2

License: (c) (i) This work is licensed under a Creative Commons Attribution 4.0 International License. Read Full License 
Page 2/16 


\section{Abstract}

Subarachnoid hemorrhage ( $\mathrm{SAH})$ is a devastating cerebral vascular disease which causes neurological deficits including long-term cognitive deficit. Demyelination of white matter is correlated with cognitive deficit in SAH. Electroacupuncture $(E A)$ is a traditional Chinese medical treatment which protects against cognitive deficit in varies of neurological diseases. However, whether EA exerts protective effect on cognitive function in SAH has not been investigated. The underlying mechanism of remyelination regulated by EA remains unclear.This study aimed to investigate the protective effects of EA on cognitive deficit in a rat model of SAH. SAH was induced in SD rats $(n=70)$ by endovascular perforation. Rats in EA group received EA treatment (10min per day) under isoflurane anesthesia after SAH. Rats in SAH and sham groups received no treatment. The mortality rate, neurological score, cognitive function, cerebral blood flow (CBF), and remyelination in sham, SAH and EA groups were assessed at $21 \mathrm{~d}$ after SAH.EA treatment alleviated cognitive deficits and myelin injury of rats compared with that in SAH group. Moreover, EA treatment enhanced remyelination in white matter and promoted the differentiation of OPCs after SAH. EA treatment inhibited the expression of Id 2 and promoted the expression of SOX10 in oligodendrocyte cells. Additionally, the cerebral blood flow (CBF) of rats was increased by EA compared with that in SAH group. EA treatment exerts protective effect against cognitive deficit in the late phase of $\mathrm{SAH}$. The underlying mechanisms involve promoting OPC differentiation and remyelination in white matter via regulating the expression of Id2 and SOX10. The improvement of CBF may also account for the protective effect of EA on cognitive function. EA treatment is a potential therapy for the treatment of cognitive deficit after SAH.

\section{Introduction}

Subarachnoid hemorrhage (SAH) is a devastating cerebrovascular disease which induces high mortality and neurological deficits (Pang et al. 2019). Previously, most endeavors have been made focusing on the protection of neurons and grey matter, while the research progress of white matter injury in SAH remains slow. White matter occupies over $50 \%$ of volume in the human brain, which mainly consists of axons and myelin (Lipscomb et al. 1998). White matter has been shown to be more vulnerable to ischemia and hemorrhagic stroke than cortex. The integrity of white matter plays an important role in cognitive function. It is reported that white matter injury is major cause of cognitive deficits in traumatic brain injury (TBI) (Kou and VandeVord 2014). In the early phase of SAH, white matter injury is characterized by myelin basic protein (MBP) degradation, amyloid precursor protein (APP) accumulation and white matter edema (Egashira et al. 2015; Kummer et al. 2015). Moreover, our clinical observation showed that white matter abnormality occurs on DTI in the late phase of SAH, which is associated with cognitive deficit of SAH patients (Reijmer et al. 2018). However, progress of the white matter repairment and the cognitive recovery after $\mathrm{SAH}$ remains lacking.

Acupuncture, derived from traditional Chinese medicine, has been used for centuries to treat various neurological diseases (Lee et al. 2020). Modern medicine applies electrical stimulation through a small electric current passing between pairs of acupuncture needles, which is known as electro-acupuncture 
(EA) (Liu et al. 2015). EA has been widely used in the promotion of functional recovery after stroke in clinical practice (Zhang et al. 2018). Acupuncture alleviates pathological process including edema, inflammation, and apoptosis (Liu et al. 2017), and eventually improves the neurological functions after stroke. In traditional Chinese medicine, Baihui (GV20) (Zhang et al. 2018)هDazhui (DU14) (Chavez et al. 2017) 『and Zusanli (ST36) (Chavez et al. 2017) were usually chosen to treat neurological diseases. Our previous work indicates that EA exert a protective effect on myelin in a spinal cord injury model (Huang et al. 2015). However, whether EA exerts protective effect on myelin and cognitive function in SAH has not been investigated. The underlying mechanism of remyelination regulated by EA remains unclear.

We hypothesized that EA may be a potential method to treat demyelination and cognitive deficit after $\mathrm{SAH}$. In the present study, SAH model was induced in rats. The effects of EA on myelin injury and cognitive deficit as well as their underlying mechanisms were investigated in the late phase of SAH.

\section{Materials And Methods}

All procedures were approved by the Institutional Animal Care and Use Committee (IACUC) at the Chongqing Medical University.

\section{Animals and Experimental Design}

Approval and consent for our study were obtained from our institution's Animal Care and Ethics Committee. A total of 72 adult (8-10 weeks) Sprague-Dawley rats were provided by the experimental animal center of Chongqing medical university. Rats were housed in a temperature-controlled $\left(24^{\circ} \mathrm{C}\right)$ room on a 12/12 dark/night time cycle in the laboratory animal center before the experiment. Rats $(n=72)$ were randomly divided into 3 groups on each time point: (1) the sham group $(n=8)$ : rats underwent a sham operation without the perforation of the anterior cerebral artery which induces SAH. (2) the SAH group $(n=8)$ : SAH was induced and rats were sacrificed on $7 d, 14 d, 21 d$, and $28 d$ after SAH induction. (3) the EA group $(n=8)$ : rats received 10 minutes consistent EA treatment and were sacrificed on $7 d, 14 d$, 21d, and 28d after SAH induction.

\section{SAH Induction}

Induction of SAH was performed as previously described before (Feiler et al. 2010). Briefly, rats were anesthetized with $3 \%$ isoflurane in $70 / 30 \%$ medical air/oxygen by a rodent ventilator. The right common carotid artery (CCA), external carotid artery (ECA), and internal carotid artery (ICA) were exposed. A 5-0 prolene filament (Ethicon, USA) was advanced into the anterior cerebral artery (ACA) via the ECA and ICA. After a subtle resistance was encountered, the filament was advanced $2 \mathrm{~mm}$ further to perforate the ACA. Subsequently, the filament was immediately withdrawn. In the sham operation group, the same procedure was performed with the exception of the perforation of the ACA. The body temperature was maintained at $37.5 \pm 0.5^{\circ} \mathrm{C}$ during the operation. Since SAH induces a high mortality rate, mice that died within $24 \mathrm{~h}$ were excluded and relevant mice were supplemented.

\section{Electroacupuncture Treatment}


For the EA group, EA treatment was performed at Baihui (GV20), Dazhui (DU14) and Zusanli (ST36) acupoints under anaesthesia by isoflurane. Three pairs of needles were connected with the output terminals of an EA apparatus (Model G6805-2A, China). Alternating strings of dense-sparse frequencies ( $30 \mathrm{~Hz}$ for $1.05 \mathrm{~s}$ and $4 \mathrm{~Hz}$ for $2.85 \mathrm{~s}$, alternately) were used for EA. Electrical stimulation lasted for 10 min per day on each rat.

\section{Neurological Score}

Performed by an investigator blinded to group assignment, a modified Garcia's method was used to obtain neurological scores for 1d, 2d, 3d, 7d, and 28d in SAH, EA and control group (Parrar et al. 2002). Briefly, a 27-point scoring system was used to assess the neurological deficits of rat, including spontaneous activity, limb symmetry, climbing, balance, body proprioception, vibrissae sensation, and tactile sensation.

\section{Morris Water Maze}

As a test for spatial learning and memory, Morris water maze (MWM) includes cued learning procedure, spatial acquisition task, reference memory task, and working memory task according to the previous study (JEON et al. 2010). The MWM consisted of a circular pool $2 \mathrm{~m}$ in diameter and $0.75 \mathrm{~m}$ in height. It was filled with water to a depth of $0.4 \mathrm{~m}$ and kept at room temperature. Four equally spaced points were arbitrarily designated as north $(N)$, south $(S)$, east $(E)$, and west $(W)$ around the circumference of the pool. This established four quadrants (NW, NE, SE, and SW). A clear plexiglass platform $(10 \mathrm{~cm} 910 \mathrm{~cm})$ was submerged $4 \mathrm{~cm}$ below the water level in the middle of one of the eight equally spaced arbitrary lines $(\mathrm{N}$, $\mathrm{S}, \mathrm{E}, \mathrm{W}, \mathrm{NW}, \mathrm{NE}, \mathrm{SE}$, and SW). Three large unique shapes were placed on three walls to function as distal cues around the pool. A camera mounted in the center of the ceiling above the pool tracked the rat. Behavior testing was performed between 10:00 and 18:00. All animals were housed at a constant temperature of $22^{\circ} \mathrm{C}$, under a 12-h light/dark cycle, with free access to food and water.

\section{Cerebral Blood Flow Monitoring}

Cerebral blood flow (CBF) of rats in each group was monitored by PeriCam PSI System (Perimed AB, China). Briefly, an incision was made along the sagittal axis on the rat's scalp under anaesthesia by isoflurane. Then rats were placed on the stereolocator, and the skull was completely exposed to the CBF detector. The monitoring lasted for 1 minute for each rat. Then rats were executed and the brain tissue was harvested.

\section{Transmission Electron Microscopy}

Rats in each group were perfused with $4^{\circ} \mathrm{C} 150 \mathrm{ml}$ normal saline and $100 \mathrm{ml} 4 \%$ glutaraldehyde solution, and then brain samples was extracted. Each specimen was immediately fixed with $2.5 \%$ glutaraldehyde for 2 days, post-fixed in $2 \%$ osmic acid for $1 \mathrm{~h}$, dehydrated in serial alcohol and propylene oxide, and embedded in araldite for morphometric analyses on semithin sections. Ultrathin sections mounted on copper grids were stained with uranyl acetate and lead citrate. Images were obtained using transmission electron microscopy (TEM) (Hitachi, Japan). 


\section{Western Blotting}

The white matter samples of rats in sham, SAH, and EA groups were homogenized in 10 volumes of icecold RIPA (Beyotime, China) added by protease inhibitor cocktail (Roche, Indianapolis, USA). Prepared protein extracts were subjected to sodium dodecyl sulfate-polyacrylamide gel electrophoresis and transferred to polyvinylidene difluoride membranes. The membranes were probed overnight at $4{ }^{\circ} \mathrm{C}$ with the following primary antibodies: PDGFR (1:1000, Abcam, UK), GPR17 (1:1000, Abcam, UK), SOX10 (1:1000, Abcam, UK),APC(1:1000, Abcam, UK) GAPDH (1:1000, Abcam, UK), PLP (1:1000, Abcam, UK), $\operatorname{MBP}(1: 1000, A b c a m, U K)$, and ID2 (1:1000, Abcam, UK) followed by incubation with secondary antibodies conjugated with horseradish peroxidase(1:10000, Beyotime, China). The bands were revealed using an ECL western blotting kit (Beyotime, China), and photographed by a chemiluminescence imaging system (Bio-Rad, USA). The amount of protein in each band was quantified using Image Lab software (Bio-Rad, USA).

\section{Immunofluorescence Staining}

Immunofluorescence staining was performed on frozen sections of rats in sham, SAH, and EA groups. The sections were incubated with mouse anti-rat primary antibody including Olig2 (1:250, Millipore, USA), SMI32 (1:250, Biolegend, China ), APC (1:250, Calbiochem, USA ), and rabbit anti-rat primary antibody including MBP (1:250, Abcam, USA), PDGFR-a (1:250, Abcam, USA), SOX10 (1:100, Abcam, USA), Olig2(1:250, Abcam, USA), and Id2(1:50, Genetex, USA) at $4 \circ C$ overnight. The sections were then incubated with second antibody including dylight 488-conjugated goat anti-rabbit antibody, 594conjugated goat anti-rabbit antibody, 488-conjugated goat anti-mouse antibody, and 594-conjugated goat anti-mouse antibody accordingly. DAPI was used for nuclear staining.

\section{Statistical Analysis}

All data was expressed as the mean \pm standard deviation (SD) and compared with repeated measures of analysis of variance (ANOVA). Bonferroni's post hoc method was applied for comparison among groups. The difference of mortality rate was analyzed using chi-square test. All statistic values were calculated using SPSS 28.0 (SPSS, USA). Significance was assumed at $P<0.05$.

\section{Results}

\section{Mortality and Neurological Score}

No rat died in sham operated group. The mortality rates of SAH group were $50.00 \%, 12.5 \%, 14.29 \%$, and $28.57 \%$ on $7 d, 14 d, 21 d, 28 d$, respectively after SAH. While the mortality rates of EA group were $55.56 \%$, $25.00 \%, 7.69 \%, 10.00 \%$, respectively after SAH. However, there was no significant difference in mortality between the SAH and EA groups at the same time points (Figure 1A).

The Garcia Score reflected a comprehensive neurological function in rats. The neurological scores of control group were $20.80 \pm 0.45,20.80 \pm 0.45,20.80 \pm 0.55,19.80 \pm 0.84$, on $1 d, 2 d, 3 d$, and $7 d$, 
respectively. SAH induced a sharp decrease of Garcia Score to $13.75 \pm 2.36$ on $1 \mathrm{~d}\left({ }^{\star} P<0.05\right.$, compared to sham), $15.5 \pm 2.64$ on $2 \mathrm{~d}\left({ }^{*} P<0.05\right.$, compared to sham), and $15.25 \pm 1.26$ on $3 \mathrm{~d}\left({ }^{*} P<0.05\right.$, compared to sham) compared to the sham group. The Garcia scores of EA group were $16.67 \pm 0.58$ on $1 \mathrm{~d}\left({ }^{\#} P<0.05\right.$, compared to SAH), $18.33 \pm 2.08$ on $2 \mathrm{~d}\left({ }^{\#} P<0.05\right.$, compared to $\left.S A H\right)$ and $17.33 \pm 1.15$ on $3 \mathrm{~d}\left({ }^{\#} P<0.05\right.$, compared to $\mathrm{SAH}$ ), indicating a significant improvement of Garcia score by EA treatment in the early phase of SAH. However, when it came to 7d, there was no significant difference between the control, SAH and EA groups (Figure 1B).

\section{EA Alleviated Cognitive Deficit after SAH}

Morris water maze was performed to evaluate the cognitive deficit in rats after $\mathrm{SAH}$. The results showed that rats in SAH group spent significantly longer time to reach the platform on $21 \mathrm{~d}\left({ }^{*} P<0.05\right.$, compared to sham), and rats in SAH group had a significantly greater distance moved from the target $\left({ }^{*} P<0.05\right.$, compared to sham) compared with the sham group (Figure 1C). EA treatment decreased the latency ( ${ }^{\#} P<$ 0.05 , compared to SAH) (Figure 1D) as well as reduced the distance moved for platform of rats $\left({ }^{\#} P<0.05\right.$, compared to SAH) compared to the rats in SAH group (Figure 1E).

\section{EA Promoted Remyelination after SAH}

The expression of MBP and SMI32 were revealed by immunofluorescence in the white matter. SAH induced a decrease of MBP and an increase of SMI32, while EA treatment preserved the volume of MBP and decreased the expression of SMI32 in the white matter (Figure 2A). We investigated the ultrastructural features of myelinated nerves of the white matter by TEM. The myelin in SAH groups showed varied degrees of swelling, and the cell organelles in the axoplasm became degenerative or disintegrated. Myelin sheaths became edematous, and the layers of myelin sheaths were disordered, thickened, and broken down in SAH group compared to the sham. By contrast, the degree of myelin swelling in the EA group was milder than that in the SAH group (Figure 2B). For quantitative analysis, the expression of PLP and MBP was detected by western blotting. The results showed that SAH reduced the expression of PLP and MBP ( ${ }^{\star} P<0.05$, compared to sham), while EA treatment increased the expression of these myelin structure proteins ( ${ }^{P} P<0.05$, compared to $\mathrm{SAH}$ ) (Figure $2 \mathrm{C}$ ). These results suggested that EA treatment promoted remyelination, which may explain the improvement of cognitive function in rats by EA in the late phase of SAH.

\section{EA Promoted OPC Differentiation after SAH}

To investigate the mechanism of increased remyelination after EA treatment, we detected the expression of OPC and mature oligodendrocyte markers by western blotting. The results showed that SAH induced an increase of PDGFR-a $\left({ }^{*} P<0.05\right.$, compared to sham) and a decrease of APC $\left({ }^{\star} P<0.05\right.$, compared to sham). In contrast, EA treatment decreased the expression of PDGFR-a ( ${ }^{\#} P<0.05$, compared to SAH) and increase the expression of APC ( ${ }^{\#} P<0.05$, compared to SAH) after SAH (Figure 3). Immunofluorescence staining proved the results of western blotting. SAH increased the number of OPCs while EA treatment 
revered the trend in the white matter (Figure $4 \mathrm{~A})$. The number of mature oligodendrocytes decreased after $\mathrm{SAH}$ while EA treatment increased the number of them (Figure 4B). These results indicated that SAH inhibited the differentiation of OPC and the maturation of oligodendrocytes, while EA treatment reversed the pathological process, hence promoted remyelination after SAH.

To further investigate the mechanism of EA on the modulation of OPC differentiation, we detected the expression of key transcription factors in myelination. We previously found that EA promoted Olig2 and attenuated Id2 expression in a rat model of spinal cord injury (Huang et al., 2015). In the present work, the expression of Olig2, Id2, and SOX10 were detected. The results showed that there was a decrease in the expression of Olig2 $\left({ }^{\star} P<0.05\right.$, compared to sham) and SOX10 ( ${ }^{\star} P<0.05$, compared to sham), while the expression of Id 2 increased ( ${ }^{*} P<0.05$, compared to sham) detected by western blotting after SAH (Figure 3). EA treatment inhibited the expression of $\mathrm{Id} 2\left({ }^{\#} P<0.05\right.$, compared to $\left.\mathrm{SAH}\right)$ and increased the level of SOX10 ( ${ }^{\#} P<0.05$, compared to SAH). However, EA did not change the expression of Olig2 after SAH (Figure 3). Co-staining of Olig2 with Id2 or SOX10 exhibited that the expression of Id2 elevated in oligodendrocyte cells after SAH and was inhibited by EA treatment (Figure 4C). SAH downregulated the expression of SOX10 while EA treatment upregulated the expression of SOX10 in the oligodendrocyte cells (Figure 4D). These results indicated that modulation of Id2 and SOX10 may be involved in the promotion of OPC differentiation by EA treatment after $\mathrm{SAH}$.

\section{EA Improved CBF after SAH}

The CBF of rats in each group was monitored by laser speckle method. The results showed that there was a significant decrease of CBF in rat brain after SAH compared to the sham $\left({ }^{*} P<0.05\right.$, compared to sham). However, the CBF of rats in EA group showed a significant increase on $21 \mathrm{~d}$ and $28 \mathrm{~d}$ compared to that in the SAH group ( ${ }^{\#}<<0.05$, compared to $\mathrm{SAH}$ ) (Figure 5).

\section{Discussion}

In the present study, experimental SAH induced evident mortality and cognitive deficit in rats. EA treatment preserved cognitive function, and promoted remyelination in white matter in the late phase of SAH. Differentiation of OPCs and maturation of oligodendrocytes were promoted by EA. Moreover, the expression of Id2 was downregulated and SOX10 was downregulated by EA in the oligodendrocyte cells after $\mathrm{SAH}$. The results showed that the attenuation of cognitive deficit by EA treatment after SAH may rely on the promoted remyelination in white matter. The mechanisms involve the modulation on the differentiation of OPCs and the regulation of transcription factor expression by EA treatment. Moreover, the improvement of CBF was observed in EA treatment rats, which may contribute to the protective effects of EA on the myelin.

Previous studies usually focus on the protection of gray matter and neurons in SAH, whereas white matter injury was usually overlooked. Recent studies have revealed that SAH induces white matter injury which includes the injury of axons and myelin sheath (Egashira et al. 2015; Kummer et al. 2015). In our 
previous work, white matter injury and demyelination were also observed in the early phase of SAH (Wu et al. 2015). However, the acute damage of myelin in SAH is difficult for prevention and treatment. Moreover, our previous clinical study observed the correlation between white matter injury and cognitive deficit in the long-term follow up after SAH (Darwazeh et al. 2018). Remyelination is inhibited in the late phase of $\mathrm{SAH}$. In the present work, we observed that the motor function of rats has recovered to the level of shamoperated ones on day 7 after SAH, while the cognitive deficit was observed throughout the late stage of $\mathrm{SAH}$. Therefore, more attention needs to be paid in the recovery of neurological function and the remyelination in the late stage of $\mathrm{SAH}$.

Recent work has revealed that oligodendrocytes and myelin play crucial roles in learning and memory (Xin and Chan 2020). Speed of pulse conduction in myelinated axons which is associated with cognitive function is closely related to intact structure of myelin. The thickness of myelin sheath relative to the diameter of the wrapped axon (g-ratio) and internode length and spacing are main factors of myelin plasticity. An additional, crucial function of myelin-forming oligodendrocytes provide metabolic support to the ensheathed axons (Monje 2018). In physiological conditions, decreased fractional anisotropy (FA) in white matter correlates with poor learning ability (Keller and Just 2009). Under pathological conditions, demyelination is strongly correlated to cognitive deficit. Multiple sclerosis is a typical demyelinating neurological disease, in which significant decline in cognitive function is related to demyelination (Eijlers et al. 2018). Myelin degeneration and diminished myelin renewal contribute to age-related impairment of memory (Wang et al. 2020). Imaging study also provides evidence of demyelination in cognitive impairment and dementia patient, which is characterized as decrease of myelin content (Bouhrara et al. 2018). On the contrary, remyelination benefits cognitive recovery in neurological diseases. Chen et al. proved that enhancing myelin renewal reverses cognitive dysfunction in a murine model of Alzheimer's disease (Chen et al. 2021). Transplantation of OPCs is found to promotes remyelination and rescue cognitive function in radiation induced cognitive deficit (Piao et al. 2015). Therefore, promoting remyelination is a potential method for the recovery of intact neuronal function for the treatment of cognitive deficit in SAH.

Electroacupuncture is a traditional Chinese medical therapy which has been used in treating various of neurological diseases. The underlying mechanisms of EA's curative effects are complicated. In recent study, EA is found to adjust body function through stimulating peripheral sensory nerve receptor distributing in specific areas (Liu et al. 2021). Our previous work showed that EA exerts protective effects on myelin sheath via promotion of oligodendrocyte proliferation and inhibition of oligodendrocyte death in a rat model of spinal cord injury (Huang et al. 2015). In the present study, the acupoints of Baihui (GV20) and Dazhui (GV14) were selected and were found to exert protective effect on myelin. It is previously reported that EA stimulation on Baihui (GV20) and Dazhui (GV14) enhances neurological recovery and reduces hemorrhage volume via anti-apoptosis in a model of intracerebral hemorrhage (Zhu et al. 2017). Specifically, EA is observed to exert particular therapeutic effect on cognitive deficit. Wang et al. reported that EA improves learning and memory functions in a rat model of ischemia and reperfusion cerebral injury through activation of PI3K/Akt signaling pathway (Wang et al. 2021). Other mechanisms relating to the protective effect of EA on cognitive function involve improvement of impaired long-term 
potentiation (LTP) (Chavez et al. 2017), restoration of hippocampal synaptic plasticity (Han et al. 2021), and inhibition of neuroinflammation (Cai et al. 2019). In the present study, promotion of remyelination by EA was firstly observed in SAH and may provide new evidence for the treatment of cognitive deficit.

The recovery of integrity of neuronal functions relies on the remyelination of axons in SAH. Remyelination is based on two major processes which are the differentiation of OPCs and the subsequent maturation of oligodendrocytes (Hubler et al. 2018; Kuhn et al. 2019; Pu et al. 2018). In the present study, we observed that EA enhanced the differentiation of OPCs as well as increased the number of mature oligodendrocytes in white matter after $\mathrm{SAH}$, which indicates that EA promotes the both processes of remyelination. Previous studies have showed that EA promotes proliferation (Huang et al. 2015; Li et al. 2010) and differentiation (Li et al. 2010) of OPCs in a rat model of spinal cord injury. Huang et al. found that EA treatment promotes remyelination in the demyelinated spinal cord and improves the neurological outcome (Xin and Chan. 2020). These studies indicate that EA treatment is a promising method for the enhancement of remyelination. Specifically, promotion of remyelination by EA improves cognitive outcome. It is reported that EA promotes memory recovery following white matter injury through promoting oligodendrocyte regeneration via the NT4/5-TrkB signaling pathway (Ahn et al. 2016). Our previous work found that EA exerts protective effects on myelin via modulating the interaction between Olig2 and Id2 (Ross et al. 2003). The modulatory effect of EA on remyelination was widely proved in neurological diseases. However, the detailed molecular mechanisms are still need investigating.

Transcriptional control of myelination and remyelination in physiological and pathological conditions is regulated by a complex network of transcriptional factors (Sock and Wegner 2019). Wherein Olig2 and Id2 are key factors in remyelination process. Olig2 plays positive roles in pattern formation and generation of OPCs and motor neurons (Buffo et al. 2005), and Olig2-positive OPCs are deemed as specific precursor cells for oligodendrocyte development. On the other hand, Id2 is a member of the inhibitor of differentiation family which negatively modulate the proliferation of OPCs and the development of oligodendrocytes (Jessen and Mirsky 2008). In the present study, we observed that EA inhibited the expression of PDGFR-a, the marker of OPCs, as well as decreased the number of Olig2 positive-OPCs which are the specific precursor cells of oligodendrocyte in the late phase of SAH. Moreover, EA promoted the expression of APC, the marker of mature oligodendrocytes, as well as increased the number of Olig2/APC double-staining cells, which demonstrated that EA promoted the maturation of oligodendrocytes. These results indicates that EA facilitates remyelination via promoting the OPC differentiation and oligodendrocyte maturation. Id2 works as a negative regulate transcriptive factor of oligodendrocyte by enhancing proliferation and slowing differentiation (Huang et al. 2015; Chen et al. 2012). Under physiological condition, SOX10 is a transcription factor that is required for lineage progression, terminal differentiation and the induction of myelination (Hornig et al. 2013). In the present study, we observed that EA inhibited the expression of Id2 and upregulated the expression of SOX10, indicating that EA possesses the effect of modulating the transcription factor expression in OPC differentiation, which may explain the promoting effect of EA on remyelination. 
Due to the deep location of white matter in the brain (Ulloa et al. 2017), the blood flow in the white matter is usually less than that in the cortex, which determines that white matter is more vulnerable to cerebral hypoperfusion. Our previous work observed that the blood flow decreased dramatically in white matter in SAH patients (Peng et al. 2017). Therefore, improving the CBF is a promising therapeutic method for the treatment of white matter injury. Ma et al. discovered that acupuncture increased the $\mathrm{CBF}$, and preserved the myelin integrity without influencing the axons in a rat model of vascular dementia (Ma et al. 2020). In a clinic trial, EA treatment increased CBF and improved the outcome of SAH patients through alleviating vasospasm (Lee et al. 2020). Lee et al. reported that EA modulates NO and ET-1 levels of plasma after SAH (Lee et al. 2020). Schmahmann et al. found that EA ameliorates cerebral ischemia through antiinflammatory effects (Ulloa et al. 2017). These studies may explain the effect of EA on improving the CBF in SAH. The present study observed a significant increase of blood flow on day 21 and day 28 after SAH, indicating that EA exerts a long-term improvement of $\mathrm{CBF}$ in $\mathrm{SAH}$ pathological process, which may also explain the protective effect of EA on white matter on the late stage of SAH.

\section{Conclusion}

In summary, the present study revealed that SAH induced myelin injury and cognitive deficit in rats, while EA promoted remyelination and improved cognitive function after SAH probably via promoting the differentiation of OPCs and remyelination of axons. The underlying mechanism may involve the regulation of the expression of Id2 and SOX10. Moreover, the improvement of CBF by EA was observed, which may also may explain the protective effect of EA on white matter. EA may be a potential treatment for cognitive deficit in the late phase of SAH.

\section{Declarations}

Acknowledgements We grant our gratitude to Siqin Huang(Traditional Chinese Medicine College, Chongqing Medical University, Chongqing, China) and her team for their help of instructing us to use electroacupuncture.

Data Availability Statement The data that support the findings of this study are not openly available due to privacy protection and are available from the corresponding author upon reasonable request .

Author's ContributionYW, XCS, SQS, and ZDG contributed the conception and design of the study. YYW, $X M Y, Y C C, X G L, R X$ and JY organized the database. $Y Y W$ and $X M Y$ performed the statistical analysis. YYW and XMY wrote the first draft of the manuscript. YYW, XMY, YCC, XGL, RX, and JY wrote the sections of the manuscript. YW and XCS contributed to manuscript revision, read and approved the submitted version. All authors read and approved the final manuscript.

Funding Information This work was supported by the National Natural Science Foundation of China (No.81901210 No.82071332 and No.82071397) and the Science and Technology Research Program of Chongqing Municipal Education Commission (Grant No.KJQN202000428). 
Conflict of Interest The authors declare that they have no conflicts of interest.

Ethicals approval All procedures were approved by the Institutional Animal Care and Use Committee (IACUC) at the Chongqing Medical University.

Consent to participate The informed consent forms were obtained from the participants

Consent for publication All authors discussed the results and commented on the manuscript. Neither the article nor portions of it have been previously published elsewhere. The manuscript is not under consideration for publication in another journal, and will not be submitted elsewhere until the "Metabolic Brain Disease" editorial process is completed, and all authors consent to the publication of the manuscript in "Metabolic Brain Disease".

\section{References}

1. Ahn SM, Kim YR, Kim HN, Shin YI, Shin HK, Choi BT. Electroacupuncture ameliorates memory impairments by enhancing oligodendrocyte regeneration in a mouse model of prolonged cerebral hypoperfusion. Sci Rep. 2016;6:28646. Published 2016 Jun 28. doi:10.1038/srep28646

2. Buffo A, Vosko MR, Ertürk D, et al. Expression pattern of the transcription factor Olig2 in response to brain injuries: implications for neuronal repair. Proc Natl Acad Sci U S A. 2005;102(50):18183-18188. doi:10.1073/pnas.0506535102

3. Bouhrara M, Reiter DA, Bergeron CM, et al. Evidence of demyelination in mild cognitive impairment and dementia using a direct and specific magnetic resonance imaging measure of myelin content. Alzheimers Dement. 2018;14(8):998-1004. doi:10.1016/j.jalz.2018.03.007

4. Cai M, Lee JH, Yang EJ. Electroacupuncture attenuates cognition impairment via antineuroinflammation in an Alzheimer's disease animal model. J Neuroinflammation. 2019;16(1):264. Published 2019 Dec 13. doi:10.1186/s12974-019-1665-3

5. Chavez LM, Huang SS, MacDonald I, Lin JG, Lee YC, Chen YH. Mechanisms of Acupuncture Therapy in Ischemic Stroke Rehabilitation: A Literature Review of Basic Studies. Int J Mol Sci. 2017;18(11):2270. Published 2017 Oct 28. doi:10.3390/ijms18112270

6. Chen JF, Liu K, Hu B, et al. Enhancing myelin renewal reverses cognitive dysfunction in a murine model of Alzheimer's disease. Neuron. 2021;109(14):2292-2307.e5.

doi:10.1016/j.neuron.2021.05.012

7. Chen S, Feng H, Sherchan P, et al. Controversies and evolving new mechanisms in subarachnoid hemorrhage. Prog Neurobiol. 2014;115:64-91. doi:10.1016/j.pneurobio.2013.09.002

8. Chen XS, Zhang YH, Cai QY, Yao ZX. ID2: A negative transcription factor regulating oligodendroglia differentiation. J Neurosci Res. 2012;90(5):925-932. doi:10.1002/jnr.22826

9. Darwazeh R, Wei M, Zhong J, et al. Significant Injury of the Mammillothalamic Tract without Injury of the Corticospinal Tract After Aneurysmal Subarachnoid Hemorrhage: A Retrospective Diffusion Tensor Imaging Study. World Neurosurg. 2018;114:e624-e630. doi:10.1016/j.wneu.2018.03.042 
10. Eijlers AJC, van Geest Q, Dekker I, et al. Predicting cognitive decline in multiple sclerosis: a 5-year follow-up study. Brain. 2018;141(9):2605-2618. doi:10.1093/brain/awy202

11. Han X, Gao Y, Yin X, et al. The mechanism of electroacupuncture for depression on basic research: a systematic review [published correction appears in Chin Med. 2021 Feb 10;16(1):20]. Chin Med. 2021;16(1):10. Published 2021 Jan 13. doi:10.1186/s13020-020-00421-y

12. Hornig, J., Frob, F., Vogl, M. R., Hermans-Borgmeyer, I., Tamm, E. R., \& € Wegner, M. (2013). The transcription factors Sox 10 and Myrf define an essential regulatory network module in differentiating oligodendrocytes. PLoS Genet, 9, e1003644.

13. Huang S, Tang C, Sun S, et al. Protective Effect of Electroacupuncture on Neural Myelin Sheaths is Mediated via Promotion of Oligodendrocyte Proliferation and Inhibition of Oligodendrocyte Death After Compressed Spinal Cord Injury. Mol Neurobiol. 2015;52(3):1870-1881. doi:10.1007/s12035014-9022-0

14. Hubler Z, Allimuthu D, Bederman I, et al. Accumulation of 8,9-unsaturated sterols drives oligodendrocyte formation and remyelination. Nature. 2018;560(7718):372-376. doi:10.1038/s41586-018-0360-3

15. Jessen KR, Mirsky R. Negative regulation of myelination: relevance for development, injury, and demyelinating disease. Glia. 2008;56(14):1552-1565. doi:10.1002/glia.20761

16. JEON H, Al J, SABRI $M$ et al. Learning deficits after experimental subarachnoid hemorrhage in rats. Neuroscience 2010; 169:1805-1814.

17. Keller TA, Just MA. Altering cortical connectivity: remediation-induced changes in the white matter of poor readers. Neuron. 2009;64(5):624-631. doi:10.1016/j.neuron.2009.10.018

18. Kou Z, VandeVord PJ. Traumatic white matter injury and glial activation: from basic science to clinics. Glia. 2014;62(11):1831-1855. doi:10.1002/glia.22690

19. Kuhn S, Gritti L, Crooks D, Dombrowski Y. Oligodendrocytes in Development, Myelin Generation and Beyond. Cells. 2019;8(11):1424. Published 2019 Nov 12. doi:10.3390/cells8111424

20. Lee DH, Cho SY, Yang SB, et al. Efficacy of Acupuncture Treatment to Prevent Cerebral Vasospasm After Subarachnoid Hemorrhage: A Double-Blind, Randomized Placebo-Controlled Trial. J Altern Complement Med. 2020;26(12):1182-1189. doi:10.1089/acm.2020.0156

21. Lipscomb DC, Gorman LG, Traystman RJ, Hurn PD. Low molecular weight iron in cerebral ischemic acidosis in vivo. Stroke. 1998;29(2):487-493. doi:10.1161/01.str.29.2.487

22. Li Q, Brus-Ramer M, Martin JH, McDonald JW. Electrical stimulation of the medullary pyramid promotes proliferation and differentiation of oligodendrocyte progenitor cells in the corticospinal tract of the adult rat. Neurosci Lett. 2010;479(2):128-133. doi:10.1016/j.neulet.2010.05.043

23. Liu AJ, Li JH, Li HQ, et al. Electroacupuncture for Acute Ischemic Stroke: A Meta527 Analysis of Randomized Controlled Trials. The American journal of Chinese medicine 528 2015;43(8):1541-66.

24. Liu H, Sun X, Zou W, Leng M, Zhang B, Kang X, He T, Wang H. Scalp acupuncture attenuates neurological deficits in a rat model of hemorrhagic stroke. Complement Ther Med. 2017a;32:85-90. 
25. Liu S, Wang Z, Su Y, et al. A neuroanatomical basis for electroacupuncture to drive the vagal-adrenal axis. Nature. 2021;598(7882):641-645. doi:10.1038/s41586-021-04001-4

26. Ma SM, Wang L, Su XT, et al. Acupuncture Improves White Matter Perfusion and Integrity in Rat Model of Vascular Dementia: An MRI-Based Imaging Study. Front Aging Neurosci. 2020;12:582904. Published 2020 Nov 23. doi:10.3389/fnagi.2020.582904

27. Monje M. Myelin Plasticity and Nervous System Function. Annu Rev Neurosci. 2018;41:61-76. doi:10.1146/annurev-neuro-080317-061853

28. Parra, M.J. McGirt, H. Sheng, D.T. Laskowitz, R.D. Pearlstein, D.S. Warner, Mouse model of subarachnoid hemorrhage associated cerebral vasospasm: methodological analysis, Neurol. Res. 24 (2002) 510-516.

29. Pang J, Peng J, Yang P, et al. White Matter Injury in Early Brain Injury after Subarachnoid Hemorrhage. Cell Transplant. 2019;28(1):26-35. doi:10.1177/0963689718812054

30. Peng JH, Qin XH, Pang JW, et al. Apolipoprotein E $\varepsilon 4$ : A Possible Risk Factor of Intracranial Pressure and White Matter Perfusion in Good-Grade Aneurysmal Subarachnoid Hemorrhage Patients at Early Stage. Front Neurol. 2017;8:150. Published 2017 Apr 19. doi:10.3389/fneur.2017.00150

31. Piao J, Major T, Auyeung G, et al. Human embryonic stem cell-derived oligodendrocyte progenitors remyelinate the brain and rescue behavioral deficits following radiation. Cell Stem Cell. 2015;16(2):198-210. doi:10.1016/j.stem.2015.01.004

32. Pu A, Stephenson EL, Yong VW. The extracellular matrix: Focus on oligodendrocyte biology and targeting CSPGs for remyelination therapies. Glia. 2018;66(9):1809-1825. doi:10.1002/glia.23333

33. Reijmer YD, van den Heerik MS, Heinen R, et al. Microstructural White Matter Abnormalities and Cognitive Impairment After Aneurysmal Subarachnoid Hemorrhage. Stroke. 2018;49(9):2040-2045. doi:10.1161/STROKEAHA.118.021622

34. Ross SE, Greenberg ME, Stiles CD (2003) Basic helix-loop-helix factors in cortical development. Neuron 39(1):13-25

35. S. Feiler, B. Friedrich, K. Scholler, S. C. Thal, and N. Plesnila, "Standardized induction of subarachnoid hemorrhage in mice by intracranial pressure monitoring," Journal of neuroscience methods, vol. 190, no. 2, pp. 164-170, 2010.

36. Sock E, Wegner M. Transcriptional control of myelination and remyelination. Glia. 2019;67(11):21532165. doi:10.1002/glia.23636

37. T.T. Kummer, S. Magnoni, C.L. MacDonald, et al., Experimental subarachnoid haemorrhage results in multifocal axonal injury, Brain 138 (2015) 2608-2618.

38. Ulloa L, Quiroz-Gonzalez S, Torres-Rosas R. Nerve Stimulation: Immunomodulation and Control of Inflammation. Trends Mol Med. 2017;23(12):1103-1120. doi:10.1016/j.molmed.2017.10.006

39. Wang F, Ren SY, Chen JF, et al. Myelin degeneration and diminished myelin renewal contribute to agerelated deficits in memory. Nat Neurosci. 2020;23(4):481-486. doi:10.1038/s41593-020-0588-8 
40. Wang HL, Liu FL, Li RQ, et al. Electroacupuncture improves learning and memory functions in a rat cerebral ischemia/reperfusion injury model through PI3K/Akt signaling pathway activation. Neural Regen Res. 2021;16(6):1011-1016. doi:10.4103/1673-5374.300454

41. Wu Y, Peng J, Pang J, Sun X, Jiang Y. Potential mechanisms of white matter injury in the acute phase of experimental subarachnoid haemorrhage. Brain. 2017;140(6):e36. doi:10.1093/brain/awx084

42. Xin W, Chan JR. Myelin plasticity: sculpting circuits in learning and memory. Nat Rev Neurosci. 2020;21(12):682-694. doi:10.1038/s41583-020-00379-8

43. Y. Egashira, H. Zhao, Y. Hua, et al., White matter injury after subarachnoid hemorrhage: role of bloodbrain barrier disruption and matrix metalloproteinase-9, Stroke 46 (2015) 2909-2915.

44. Zhang B, Dai XH, Yu XP, et al. Baihui (DU20)-penetrating-Qubin (GB7) acupuncture inhibits apoptosis in the perihemorrhagic penumbra. Neural Regen Res. 2018;13(9):1602-1608. doi:10.4103/16735374.237123

45. Zhu Y, Deng $L$, Tang $H$, et al. Electroacupuncture improves neurobehavioral function and brain injury in rat model of intracerebral hemorrhage. Brain Res Bull. 2017;131:123-132. doi:10.1016/j.brainresbull.2017.04.003

\section{Figures}

\section{Figure 1}

Neurological function of rats in the sham, SAH and EA groups. (A) Survival rates of rats in 3 groups. (B) Garcia score of rats in 3 groups. (C) representative tracing images from the MWM test. (D) Escape latencies from MWM test in 3 groups. (D) Swimming distances from MWM test in 3 groups. ${ }^{*} P<0.05$, compared to the sham group. ${ }^{\#} P<0.05$, compared to the SAH group.

\section{Figure 2}

Remyelination in sham, SAH, and EA groups a. (A) Immunofluorescence co-staining of MBP, SMI32, and DAPI in 3 groups on 21d after SAH. (B) The ultrastructural features of myelinated nerves detected by transmission electron microscopy (TEM). (C) Expression of PLP and MBP in 3 groups detected by western blotting. ${ }^{*} P<0.05$, compared to the sham group. ${ }^{\#} P<0.05$, compared to the SAH group. Bar scale $=100 \mu \mathrm{m}$.

\section{Figure 3}


The expression of PDGFR-a, APC, Id2, and SOX10 on $21 \mathrm{~d}$ after SAH detected by western blotting. ${ }^{*} P<$ 0.05 , compared to the sham group. ${ }^{\#} P<0.05$, compared to the SAH group.

\section{Figure 4}

Fluorescence co-staining of white matter in sham, SAH, and EA groups on 21d after $\mathrm{SAH}$. (A) Co-staining of Olig2, PDGFR-a, and DAPI in 3 groups. (B) Co-staining of Olig2, APC, and DAPI in 3 groups. (C) Costaining of Olig2, Id2, and DAPI in 3 groups. (D) Co-staining of Olig2, SOX10, and DAPI in 3 groups. Bar scale $=100 \mu \mathrm{m}$.

\section{Figure 5}

Cerebral blood flow (CBF) in sham, SAH and EA groups after SAH. Upper is the representative CBF images in 3 groups on 7d, 14d, 21d, and 28d after SAH. Lower is the bar chart of CBF in 3 groups on each time point. ${ }^{*} P<0.05$, compared to the sham group. ${ }^{\#} P<0.05$, compared to the SAH group. 